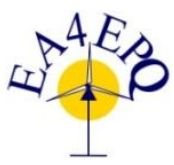

$18^{\text {th }}$ International Conference on Renewable Energies and Power Quality

(ICREPQ'20)

Granada (Spain), $1^{\text {st }}$ to $3^{\text {nd }}$ April 2020

Reneurable Ernerqy and Oomer Qualily. Fournal (RE\&PQJ)

ISSN 2172-038 X, Volume No.18, June 2020

\title{
Magnetic Saturation Impact on Three-Phase Shunt Active Power Filters
}

\author{
A. Ait Chihab ${ }^{1}$, H. Ouadi ${ }^{2}$, \\ ${ }^{1}$ PMMAT Lab, Department of Physics, Faculty of Science, University Hassan II, Casablanca (Morocco), \\ Phone: +212660529631, Email: a.aitchihab@gmail.comm \\ ${ }^{2}$ ERERA Lab. Mohammed V University ENSET Rabat (Morocco), \\ Phone: +212666247326, Email: hamidouadi3@yahoo.fr
}

\begin{abstract}
The problem of controlling three-phase shunt active power filters (SAPF) is addressed in presence of nonlinear loads. Previous works generally design control for SAPF based on standard models that assume the involved magnetic coil to be linear. In reality, the magnetic characteristics of these components are nonlinear (especially in the presence of large magnetic flux density in the ferromagnetic core). In this paper, a new oriented control model for SAPF-load system, taking into account for the nonlinearity of coil characteristics, is developed. The control objective is twofold: (i) compensating for the current harmonics and the reactive power absorbed by the nonlinear load; (ii) regulating the inverter DC capacitor voltage. To this end, based on the new model, a nonlinear controller is developed, using the backstepping technical design. It is therefore able to ensure good performances over a wide range of variation of the load current. The performances of the proposed adaptive controller are formally analyzed using tools from the Lyapunov stability and the averaging theory. The performances of the proposed controller is illustrated through simulation
\end{abstract}

Keywords. Three-phase active power filters, Magnetic saturation, Harmonics current, Reactive power, Adaptive control

\section{Introduction}

The Power grids and distribution networks are expected to simultaneously interact with a wide variety of loads. As a matter of fact, these loads (whatever their size), such as rectifiers, power supplies and speed drivers, involve nonlinear dynamics that entail the generation of current harmonics and the consumption of reactive power. If not appropriately compensated for, these current harmonics and reactive power result in several harmful effects e.g. distortion of the voltage waveform at the point of common coupling (PCC) and overheating of transformers and distribution lines. Moreover, the disturbing effect of current harmonics may go beyond the PCC and reach other loads and electronic equipments connected to the net, causing boosted ageing of those loads and making harder the synchronization with the network voltage in applications requiring such synchronization.
The modern solution to cope with harmonics pollution is to implement active power filters (APF). Indeed, compared to conventional passive filters, APFs feature a higher flexibility, a better filtering capability and a smaller physical size. There are various APF configurations but the most widely implemented in industrial scale products are the shunt configurations. The principle of shunt active power filters (SAPF) consists in injecting at the PCC a current that cancels all harmonics and reactive currents generated by the disturbing loads. In addition to this energy quality objective, there is an operational requirement that consists in regulating the DC voltage of the energy storage capacitor, placed next to the SAPF inverter. This DC voltage regulation loop control is necessary for the SAPF to work conveniently. The achievement of the above two requirements, i.e. energy quality and $\mathrm{DC}$ voltage regulation, is made difficult by the controlled system nonlinearity, on the one hand, and by the fact that some system parameters may be unknown.

Over the last decade, a great deal of interest has been devoted to the problem of controlling energy systems involving SAPFs. But, most previous works have been devoted to the simpler case of single-phase SAPFs [1]. The point is that, in industrial applications, electrical loads are generally three-phase. Many studies have examined the problem of modeling and controlling power systems that involves three-phase SAPFs [2, 3]. In these studies, the authors suppose that all passive components constituting the SAPF can be perfectly described by linear characteristics. This assumption is obviously not valid under all operational conditions, particularly for filter coils. In fact, due the saturation phenomena, the coil magnetic characteristic is non linear and consequently the inductance coefficients vary with the current, especially for high-power coils. In the other hand, the problem of controlling power systems that involves three-phase SAPFs has been dealt previously with using three categories of methods. The first category includes methods using hysteresis operators or fuzzy logics [4]. These methods do not make use of the exact nonlinear SAPF model in the control design. Consequently, the obtained controllers are generally not backed by formal stability analysis and their performances are derived from simulations results. The second category of methods is limited to linear controllers [5, 6]. As a matter of fact, optimal performances are not guaranteed with linear 
controller, on a wide range variation of the operation point, due to the nonlinear nature of the controlled system. The third category of methods includes nonlinear regulators using different control design techniques: passivity approach [7], Lyapunov design [8] and sliding mode control [9].

In this paper, we revisit the modeling of three-phase SAPF in order to account for the nonlinear feature of coil magnetic characteristics. Our model allows the inductance coefficients to vary with the corresponding coils currents according to a well-defined law. Based on this new model, a new control strategy is developed to simultaneously meet the previously discussed control requirements. To this end, a two-loop cascade nonlinear controller are developed using the Lyapunov-like techniques. The inner loop involves a current regulator designed to cope with harmonics compensation. The outer-loop involves a voltage regulator that aims at regulating the DC line voltage. These theoretical results are confirmed by numerical simulations.

The paper is organized as follows: The SAPF modeling with taking account, the nonlinear feature of the magnetic characteristic, is described in Section 2; The references signals construction and, the cascade adaptive non linear regulator design are dealt with in section 3; the theoretical analysis results are confirmed by simulation in Section 4. A conclusion and a references list end the paper.

\section{Three-phases SAPF modeling}

The three phase SAPF under study has the structure of Fig.1. It consists of a three-phase full-bridge inverter and an energy storage capacitor $C_{d c}$, placed at the DC side. From the AC side, the SAPF is connected to the network through a filtering inductor $\left(L_{f}, R_{f}\right)$; this reduces the circulation of the harmonics currents generated by the inverter.

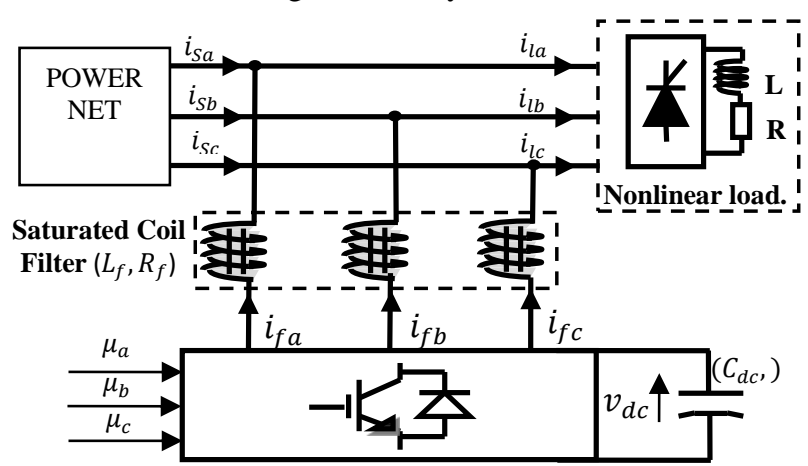

Fig.1. Structure of SAPF with ferromagnetic core

The SAPF function is to produce reactive and harmonic current components to compensate undesirable current harmonics produced by the nonlinear load. The DC-AC inverter operates in accordance to the well-known of Pulse Width Modulation principle (PWM), [10,11].

The aim of this section is to develop, for the SAPF, a control-oriented model that accounts for the saturation feature in coil magnetic cores. Many earlier works have investigated the magnetic saturation feature, especially those focusing on modeling the magnetic flux saturation in electric machines [12,13]. It has generally been captured through B-H characteristics or through flux-current characteristics: see for example [14,15]. In this paper, we follow this latter approach. Due to the saturation phenomena, the magnetic flux across the coil core is a nonlinear function of the current that is: $\emptyset_{f k}=\lambda\left(i_{f k}\right)$ where $\lambda($.$) denotes the nonlinear magnetic characteristic of the$ coil core and the subscript $\mathrm{k}$ corresponds to the phase considered.

The following notations are used for the model development:

\begin{tabular}{|l|l|}
\hline \multicolumn{1}{|c|}{ Notations } & Designation \\
\hline$v_{s a}, v_{s b}, v_{s c}$ & network voltages \\
\hline$i_{s a}, i_{s b}, i_{s c}$ & network currents \\
\hline$v_{f a}, v_{f b}, v_{f c}$ & AC inverter voltages. \\
\hline$i_{f a}, i_{f b}, i_{f c}$ & AC inverter currents. \\
\hline$\phi_{f a}, \phi_{f b}, \phi_{f c}$ & Filter coil flux \\
\hline$\lambda_{f a}, \lambda_{f b}, \lambda_{f c}$ & Magnetic characteristic of the coil \\
\hline$i_{l a}, i_{l b}, i_{l c}$ & Load current. \\
\hline$v_{s \alpha}, v_{s \beta}$ & PCC Voltage in static $\alpha-\beta$ coordinates. \\
\hline$i_{f \alpha}, i_{f \beta}$ & Output filter current in $\alpha-\beta$ coordinates. \\
\hline$\overline{l_{f \alpha}}, \overline{l_{f \beta}}$ & Outer loop control signal $(\alpha-\beta)$ \\
\hline$\widetilde{l_{f \alpha}}, \widetilde{l_{f \beta}}$ & Harmonics AC inverter current \\
\hline$u_{a}, u_{b}, u_{c}$ & PWM inverter control voltage \\
\hline$u_{\alpha}, u_{\beta}$ & PWM inverter control in $(\alpha-\beta)$ \\
\hline$V_{d c}$ & DC bus voltage \\
\hline$L_{f}$ & filter inductor \\
\hline$R_{f}$ & filter resistor \\
\hline$C_{d c}$ & DC bus capacitor. \\
\hline$R_{d c}$ & $C_{d c}$ leak resistance. \\
\hline$S_{i j}$ & Switches inverter components \\
\hline
\end{tabular}

For each phase of the SAPF, the coil voltage is given by:

$v_{L_{f}}=\frac{d \phi_{f k}}{d t}=\frac{d \lambda\left(i_{f k}\right)}{d i_{f k}} \frac{d i_{f k}}{d t}=L_{f}\left(i_{f k}\right) \frac{d i_{f k}}{d t}$

with

$L_{f}\left(i_{f k}\right) \stackrel{\text { def }}{=} \frac{d \lambda\left(i_{f k}\right)}{d i_{f k}}$

Now, applying the usual electric laws to the three-phase shunt APF, one easily gets:

$\frac{d}{d t}\left[L_{F}\left(i_{f a b c}\right) i_{f a b c}(t)\right]=-R_{f}\left[i_{f a b c}(t)\right]+\left[v_{f a b c}(t)\right]-$

$\left[v_{s a b c}(t)\right]$

On the other hand, we recall that, in the ideal case (ideals commutations of the converter switches components), the output voltages and output currents of the DC-AC inverter are respectively given by: [11]

$\left[\begin{array}{l}v_{f a}(t) \\ v_{f b}(t) \\ v_{f c}(t)\end{array}\right]=\frac{v_{d c}}{6}\left[\begin{array}{rrr}2 & -1 & -1 \\ -1 & 2 & -1 \\ -1 & -1 & 2\end{array}\right]\left[\begin{array}{l}\mu_{a}(t) \\ \mu_{b}(t) \\ \mu_{c}(t)\end{array}\right]$
$C_{d c} \frac{d v_{d c}}{d_{t}}=\frac{-1}{2}\left(\mu_{a} i_{f a}+\mu_{b} i_{f b}+\mu_{c} i_{f c}\right)-\frac{v_{d c}}{R_{d c}}$

Where the inverter switching functions $\mu_{i}(i=a, b$ or $c)$ are defined by:

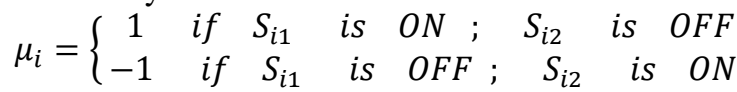

In the $(\alpha-\beta)$, reference frame, equations (4) and (5) becomes: 


$$
\begin{aligned}
& {\left[\begin{array}{l}
v_{f \alpha} \\
v_{f \beta}
\end{array}\right]=\frac{v_{d c}}{2}\left[\begin{array}{l}
u_{\alpha} \\
u_{\beta}
\end{array}\right]} \\
& \frac{d v_{d c}}{d t}=\frac{-1}{2 C_{d c}}\left(\mu_{\alpha} i_{f \alpha}+\mu_{\beta} i_{f \beta}\right)-\frac{v_{d c}}{R_{d c} C_{d c}}
\end{aligned}
$$

Similarly, using (7) and by applying the Concordia transformation to the filter equation (3), one gets:

$$
\begin{array}{r}
C_{23}\left(\begin{array}{ccc}
L_{f}\left(i_{f a}\right) & 0 & 0 \\
0 & L_{f}\left(i_{f b}\right) & 0 \\
0 & 0 & L_{f}\left(i_{f c}\right)
\end{array}\right) C_{32} \frac{d}{d t}\left[\begin{array}{l}
i_{f \alpha} \\
i_{f \beta}
\end{array}\right] \\
+C_{23}\left(\begin{array}{ccc}
\frac{d L_{f}\left(i_{f a}\right)}{d t} & 0 & 0 \\
0 & \frac{d L_{f}\left(i_{f b}\right)}{d t} & 0 \\
0 & 0 & \frac{d L_{f}\left(i_{f c}\right)}{d t}
\end{array}\right) C_{32}\left[\begin{array}{l}
i_{f \alpha} \\
i_{f \beta}
\end{array}\right]=-R_{f}\left[\begin{array}{l}
i_{f \alpha} \\
i_{f \beta}
\end{array}\right]+ \\
\frac{1}{2} v_{d c}\left[\begin{array}{l}
\mu_{\alpha} \\
\mu_{\beta}
\end{array}\right]-\left[\begin{array}{l}
v_{s \alpha} \\
v_{s \beta}
\end{array}\right]
\end{array}
$$

Where $\mathrm{C}_{32}$ and $C_{23}$ are the well known Concordia matrixes. Equation (9) can be rewritten as the more compact form:

$\left(\begin{array}{ll}h_{11}\left(i_{f \alpha}, i_{f \beta}\right) & h_{12}\left(i_{f \alpha}, i_{f \beta}\right) \\ h_{21}\left(i_{f \alpha}, i_{f \beta}\right) & h_{22}\left(i_{f \alpha}, i_{f \beta}\right)\end{array}\right) \frac{d}{d t}\left[\begin{array}{l}i_{f \alpha} \\ i_{f \beta}\end{array}\right]+$

$\left(\begin{array}{ll}g_{11}\left(i_{f \alpha}, i_{f \beta}\right) & g_{12}\left(i_{f \alpha}, i_{f \beta}\right) \\ g_{21}\left(i_{f \alpha}, i_{f \beta}\right) & g_{22}\left(i_{f \alpha}, i_{f \beta}\right)\end{array}\right)\left[\begin{array}{l}i_{f \alpha} \\ i_{f \beta}\end{array}\right]=-R_{f}\left[\begin{array}{l}i_{f \alpha} \\ i_{f \beta}\end{array}\right]+\frac{v_{d c}}{2}\left[\begin{array}{l}u_{\alpha} \\ u_{\beta}\end{array}\right]-\left[\begin{array}{l}v_{s \alpha} \\ v_{s \beta}\end{array}\right](10)$

The SAPF model, taking into account the saturation of the filter coil is therefore in the form:

$$
\begin{aligned}
& H\left(i_{f \alpha}, i_{f \beta}\right) \frac{d}{d t}\left[\begin{array}{l}
i_{f \alpha} \\
i_{f \beta}
\end{array}\right]+G\left(i_{f \alpha}, i_{f \beta}\right)\left[\begin{array}{l}
i_{f \alpha} \\
i_{f \beta}
\end{array}\right]=-R_{f}\left[\begin{array}{l}
i_{f \alpha} \\
i_{f \beta}
\end{array}\right]+ \\
& \frac{v_{d c}}{2}\left[\begin{array}{l}
\mu_{\alpha} \\
\mu_{\beta}
\end{array}\right]-\left[\begin{array}{l}
v_{s \alpha} \\
v_{s \beta}
\end{array}\right] \\
& \frac{d v_{d c}}{d t}=\frac{-1}{2 C_{d c}}\left(\mu_{\alpha} i_{f \alpha}+\mu_{\beta} i_{f \beta}\right)-\frac{v_{d c}}{R_{d c} c_{d c}}
\end{aligned}
$$

The equations (11-12) are useful for building up an accurate simulator of the SAPF. However, it cannot be based upon in the control design as it involves a binary control input, namely $\left(\mu_{\alpha}, \mu_{\beta}\right)$. This kind of difficulty is generally coped with by resorting to average models. Signal averaging is performed over cutting intervals [11].

Where the nonlinear matrix functions $\mathrm{H}\left(i_{f \alpha}, i_{f \beta}\right)=$ $\left(\begin{array}{ll}\mathrm{h}_{11}\left(x_{1}, x_{2}\right) & \mathrm{h}_{12}\left(x_{1}, x_{2}\right) \\ \mathrm{h}_{21}\left(x_{1}, x_{2}\right) & \mathrm{h}_{22}\left(x_{1}, x_{2}\right)\end{array}\right) \quad$ and $\quad G\left(i_{f \alpha}, i_{f \beta}\right)=$ $\left(\begin{array}{ll}\mathrm{g}_{11}\left(x_{1}, x_{2}\right) & \mathrm{g}_{12}\left(x_{1}, x_{2}\right) \\ \mathrm{g}_{21}\left(x_{1}, x_{2}\right) & \mathrm{g}_{22}\left(x_{1}, x_{2}\right)\end{array}\right)$

With (11), the obtained average model is the following:

$\left[\begin{array}{l}\dot{x}_{1} \\ \dot{x}_{2}\end{array}\right]=H^{-1}\left(x_{1}, x_{2}\right)\left(-\left(G\left(x_{1}, x_{2}\right)+R_{f} I_{2}\right)\left[\begin{array}{l}x_{1} \\ x_{2}\end{array}\right]+\right.$

$\left.\frac{V_{d c}}{2}\left[\begin{array}{l}u_{\alpha} \\ u_{\beta}\end{array}\right]-\left[\begin{array}{l}v_{s \alpha} \\ v_{s \beta}\end{array}\right]\right)$

where $x_{1}, x_{2}, V_{d c}, u_{\alpha}$ and $u_{\beta}$ denote the average values, over cutting periods, of the signals $i_{f \alpha}, i_{f \beta}, v_{d c}, \mu_{\alpha}$ and $\mu_{\beta}$, respectively. In (13), the mean value $\left(u_{\alpha}, u_{\beta}\right)$ of $\left(\mu_{\alpha}, \mu_{\beta}\right)$ turns out to be the system control input.

To carry out the DC bus voltage control, the system modeling must be completed with a third equation describing the energy stored in the capacitor $\left(E_{d c}=\right.$ $\left.\frac{1}{2} C_{d c} V_{d c}^{2}\right)$. To this end, consider the total power $\left(P_{D C}\right)$ at the DC bus:

$$
P_{D C}=-\frac{d}{d t}\left(\frac{1}{2} C_{d c} v_{d c}^{2}\right)
$$

Assuming that the filter switches are ideal, the introduction of (8) into (14) leads to:

$P_{D C}=\frac{v_{d c}}{2}\left(\mu_{\alpha} i_{f \alpha}+\mu_{\beta} i_{f \beta}\right)+\frac{v_{d c}^{2}}{R_{D C}}$

In practice, the switching losses in the power converter cannot be ignored. So the power balance, presented in (15) must be modified according to the following equation:

$P_{D C}=\frac{v_{d c}}{2}\left(\mu_{\alpha} i_{f \alpha}+\mu_{\beta} i_{f \beta}\right)+\frac{v_{d c}^{2}}{R_{D C}}+P_{s c}$

where $P_{S C}$, denotes the switching inverter losses (considered unknown).

Now let $x_{3}$ denote the averaged capacitor energy, using (16) one gets by operating the usual averaging (over cutting periods) :

$\dot{x}_{3}=\frac{-V_{d c}}{2}\left(u_{\alpha} i_{f \alpha}+u_{\beta} i_{f \beta}\right)-\frac{v_{d c}^{2}}{R_{D C}}-P_{s c}$

For convenience, the model equations (13) and (17) are rewritten altogether

$$
\begin{aligned}
& {\left[\begin{array}{l}
\dot{x}_{1} \\
\dot{x}_{2}
\end{array}\right]=H^{-1}\left(x_{1}, x_{2}\right)\left(-\left(G\left(x_{1}, x_{2}\right)+R_{f} I_{2}\right)\left[\begin{array}{l}
x_{1} \\
x_{2}
\end{array}\right]+\right.} \\
& \left.\sqrt{\frac{x_{3}}{2 C_{d c}}}\left[\begin{array}{l}
u_{\alpha} \\
u_{\beta}
\end{array}\right]-\left[\begin{array}{l}
v_{s \alpha} \\
v_{s \beta}
\end{array}\right]\right) \\
& \dot{x}_{3}=-\sqrt{\frac{x_{3}}{2 C_{d c}}}\left(u_{\alpha} x_{1}+u_{\beta} x_{2}\right)-\frac{2 x_{3}}{c_{d c} R_{d c}}-P_{s c}
\end{aligned}
$$

\section{Three-phases SAPF control design}

1) Currents references signals construction:

The load current decomposition introduces a harmonic component on the one hand, the active and reactive components on the other hand. This decomposition is needed to formulate the control objectives and design the controller. Presently, the decomposition is performed using the so-called instantaneous power technique, which enjoys a good compromise between accuracy and computational complexity [16]. Accordingly, the active and the reactive load powers can both be decomposed, when the load currents include harmonics, in a continuous component and a varying component, i.e.

$\left[\begin{array}{l}P \\ Q\end{array}\right]=\left[\begin{array}{l}\bar{P}+\tilde{P} \\ \bar{q}+\tilde{q}\end{array}\right]=\left[\begin{array}{cc}v_{s \alpha} & v_{s \beta} \\ -v_{s \beta} & v_{s \alpha}\end{array}\right]\left[\begin{array}{l}i_{l \alpha} \\ i_{l \beta}\end{array}\right]$

Solving this equation with respect to the currents, and rearranging terms, one gets the following decomposition:

$\left[\begin{array}{c}i_{l \alpha} \\ i_{l \beta}\end{array}\right]=\underbrace{\frac{1}{\Delta}\left[\begin{array}{cc}v_{s \alpha} & -v_{s \beta} \\ v_{s \beta} & v_{s \alpha}\end{array}\right]\left[\begin{array}{c}\bar{P} \\ 0\end{array}\right]}_{\text {actif component }}+\underbrace{\frac{1}{\Delta}\left[\begin{array}{cc}v_{s \alpha} & -v_{s \beta} \\ v_{s \beta} & v_{s \alpha}\end{array}\right]\left[\begin{array}{l}0 \\ \bar{q}\end{array}\right]}_{\text {reactif component }}+\underbrace{\frac{1}{\Delta}\left[\begin{array}{cc}v_{s \alpha} & -v_{s \beta} \\ v_{s \beta} & v_{s \alpha}\end{array}\right]\left[\begin{array}{l}\tilde{P} \\ \tilde{q}\end{array}\right]}_{\text {harmonic component }}(21)$

with $\Delta=v_{s \alpha}{ }^{2}+v_{s \beta}{ }^{2}$. Practically, the online computation of the power components $\tilde{P}, \tilde{q}$ and $\bar{q}$ is performed using the instantaneous power method .

The control signals generated by the outer loop regulator, denoted $\left(\overline{l_{f \alpha}}, \overline{l_{f \beta}}\right)$ serve as the desired fundamental components of the output current filter. These components are augmented with the (load current) harmonic and reactive components, next denoted $\left(i_{f \alpha}^{*}, i_{f \beta}^{*}\right)$, to constitute the final AC current references: $\left(x_{1}^{*}, x_{2}^{*}\right)$ 


$$
\begin{aligned}
& {\left[\begin{array}{l}
x_{1}^{*} \\
x_{2}^{*}
\end{array}\right]=\left[\begin{array}{l}
i_{l \alpha}^{*} \\
i_{l \beta}^{*}
\end{array}\right]+\left[\begin{array}{l}
\overline{l_{f \alpha}} \\
\overline{l_{f \beta}}
\end{array}\right]=\underbrace{\frac{1}{\Delta}\left[\begin{array}{cc}
v_{s \alpha} & -v_{s \beta} \\
v_{s \beta} & v_{s \alpha}
\end{array}\right]\left[\begin{array}{l}
\tilde{P} \\
\tilde{q}
\end{array}\right]}_{\text {harmonic component }}+\underbrace{\frac{1}{\Delta}\left[\begin{array}{cc}
v_{s \alpha} & -v_{s \beta} \\
v_{s \beta} & v_{s \alpha}
\end{array}\right]\left[\begin{array}{l}
0 \\
\bar{q}
\end{array}\right]}_{\text {reactif component }}+} \\
& \underbrace{\left[\begin{array}{l}
\overline{l_{f \alpha}} \\
\bar{l}_{f \beta}
\end{array}\right]}_{\text {outer control signal }}
\end{aligned}
$$

\section{2) Inner control loop design:}

The inner loop is designed to make the current tracking errors,

$\left[\begin{array}{l}Z_{1} \\ z_{2}\end{array}\right]=\left[\begin{array}{l}x_{1}-x_{1}^{*} \\ x_{2}-x_{2}^{*}\end{array}\right]$

as small as possible. To this end, the dynamics of these errors needs to be determined. It follows, using the model equations

(18-19), that the errors undergo the following equations:

$$
\begin{gathered}
{\left[\begin{array}{l}
\dot{z}_{1} \\
\dot{z}_{2}
\end{array}\right]=H^{-1}\left(x_{1}, x_{2}\right)\left(-\left(G\left(x_{1}, x_{2}\right)+R_{f} I_{2}\right)\left[\begin{array}{l}
x_{1} \\
x_{2}
\end{array}\right]+\right.} \\
\left.\sqrt{\frac{x_{3}}{2 C d c}}\left[\begin{array}{l}
u_{\alpha} \\
u_{\beta}
\end{array}\right]-\left[\begin{array}{l}
v_{s \alpha} \\
v_{s \beta}
\end{array}\right]\right)-\left[\begin{array}{l}
\dot{x}_{1}^{*} \\
\dot{x}_{2}^{*}
\end{array}\right]
\end{gathered}
$$

To ensure the asymptotic stability of the equilibrium $\left(z_{1}, z_{2}\right)=(0,0)$, equation (24) suggests that the control inputs $\left(u_{\alpha}, u_{\beta}\right)$ should be chosen so that:

$$
\begin{aligned}
& -\left[\begin{array}{l}
c_{1} z_{1} \\
c_{2} z_{2}
\end{array}\right]=H^{-1}\left(x_{1}, x_{2}\right)\left(-\left(G\left(x_{1}, x_{2}\right)+R_{f} I_{2}\right)\left[\begin{array}{l}
x_{1} \\
x_{2}
\end{array}\right]+\right. \\
& \left.\sqrt{\frac{x_{3}}{2 C d c}}\left[\begin{array}{l}
u_{\alpha} \\
u_{\beta}
\end{array}\right]-\left[\begin{array}{l}
v_{s \alpha} \\
v_{s \beta}
\end{array}\right]\right)-\left[\begin{array}{l}
\dot{x}_{1}^{*} \\
\dot{x}_{2}^{*}
\end{array}\right]
\end{aligned}
$$

Solving (25) with respect to $\left(u_{\alpha}, u_{\beta}\right)$ yields the following control law, which defines the inner regulator:

$\left[\begin{array}{l}u_{\alpha} \\ u_{\beta}\end{array}\right]=\sqrt{\frac{2 C_{d c}}{x_{3}}}\left(H\left(x_{1}, x_{2}\right)\left(\left[\begin{array}{l}\dot{x}_{1}^{*} \\ \dot{x}_{2}^{*}\end{array}\right]-\left[\begin{array}{l}Z_{1} \\ z_{2}\end{array}\right]\right)+\left(\left(G\left(x_{1}, x_{2}\right)+\right.\right.\right.$

$\left.\left.\left.R_{f} I_{2}\right)\left[\begin{array}{l}x_{1} \\ x_{2}\end{array}\right]\right)+\left[\begin{array}{l}v_{s \alpha} \\ v_{s \beta}\end{array}\right]\right)$

Combining equations (24) and (26) one gets the following equations describing the inner closed loop:

$\left[\begin{array}{l}\dot{z}_{1} \\ \dot{z}_{2}\end{array}\right]=-\left[\begin{array}{l}c_{1} z_{1} \\ c_{2} z_{2}\end{array}\right]$

It readily follows that:

$z_{1}(t)=z_{1}(0) e^{-c_{1} t}, z_{2}(t)=z_{2}(0) e^{-c_{2} t}$

This shows that the errors are globally exponentially vanishing, that is the objective of canceling the load current harmonics and load current reactive component is well established.

\section{Outer control loop design}

The outer loop aims at making the voltage tracking error,

$z_{3}=x_{3}-x_{3}^{*}$

as small as possible, where $x_{3}^{*}$ is the reference value of the DC bus voltage. Without loss of generality, it is assumed that $x_{3}^{*}$ as well as its first time-derivative are known and bounded.

Furthermore, following the notations previously presented, the AC filter currents verify:

$\left[\begin{array}{l}i_{f \alpha} \\ i_{f \beta}\end{array}\right]=\left[\begin{array}{l}\overline{l_{f \alpha}} \\ \overline{l_{f \beta}}\end{array}\right]+\left[\begin{array}{l}\widetilde{l_{f \alpha}} \\ \widetilde{l_{f \beta}}\end{array}\right]$

By introducing (30) and (7) into (17), the third SAPF state equation becomes:

$\dot{x}_{3}=-v_{f \alpha} \overline{l_{f \alpha}}-v_{f \beta} \overline{l_{f \beta}}-\frac{v_{d c}^{2}}{R_{D C}}-P_{s c}-v_{f \alpha} \widetilde{l_{f \alpha}}-v_{f \beta} \widetilde{l_{f \beta}}$

On the other hand, in practice state variable $x_{3}$ present a very slow dynamic (it is associated to the DC bus) then the AC current components $\left(\widetilde{l_{f \alpha}}\right)$ and $\left(\widetilde{l_{f \beta}}\right)$. Indeed, the latter are varying at harmonics load current frequency. Consequently, the control design is based on the average model, obtained form (31) letting there $\left\langle v_{f \alpha} \widetilde{l_{f \alpha}}+\right.$ $\left.v_{f \beta} \widetilde{l_{f \beta}}\right\rangle=0$

It turns out that the average DC voltage state is given by:

$\dot{x}_{3}=-v_{f \alpha} \overline{l_{f \alpha}}-v_{f \beta} \overline{l_{f \beta}}-\frac{v_{d c}^{2}}{R_{D C}}-P_{s c}$

Then using (26), (23) and (32), the time derivative of the error voltage (29) can be written as:

$\dot{z}_{3}=-\left(v_{f \alpha} \overline{l_{f \alpha}}+v_{f \beta} \overline{l_{f \beta}}\right)-\frac{2 x_{3}}{C_{d c} R_{d c}}-P_{s c}-\dot{x}_{3}^{*}(33)$

The switching-loss power $\left(P_{s c}\right)$ is seen as an unknown parameter in (33). Indeed, the latter is mainly depending on the load which presently is assumed to undergo a piecewise constant variation.

On the other hand, the quantity $\left(v_{f \alpha} \overline{l_{f \alpha}}+v_{f \beta} \overline{l_{f \beta}}\right)$ stands in (33) as a virtual control. Interestingly, this quantity is nothing other than the electric network power, denoted $P_{\text {net }}$, transmitted to control the voltage DC bus.

$$
P_{n e t}=\left[\begin{array}{ll}
v_{f \alpha} & v_{f \beta}
\end{array}\right]\left[\begin{array}{l}
\overline{l_{f \alpha}} \\
\overline{l_{f \beta}}
\end{array}\right]=v_{f \alpha} \overline{l_{f \alpha}}+v_{f \beta} \overline{l_{f \beta}}
$$

In order to obtain a stabilizing control law of the error system (29), let us introduce the following Lyapunov function candidate:

$$
V=\frac{z_{1}^{2}}{2}+\frac{z_{2}^{2}}{2}+\frac{z_{3}^{2}}{2}+\frac{1}{2 \gamma} \tilde{P}_{s c}^{2}
$$

Where $\hat{P}_{S c}$ denotes the online estimate of $P_{S c}$ and $\tilde{P}_{S C}=$ $P_{s c}-\hat{P}_{s c}$ is the corresponding estimation error; $\gamma$ is a positive design parameter. Deriving $V$ along (27) yields:

$\dot{V}=-c_{1} z_{1}^{2}-c_{2} z_{2}^{2}+z_{3}\left(-P_{n e t}-\frac{2 x_{3}}{c_{d c} R_{d c}}-P_{s c}-\dot{x}_{3}^{*}\right)+\frac{1}{\gamma} \tilde{P}_{s c} \tilde{P}_{s c}$
$\dot{V}=-c_{1} z_{1}^{2}-c_{2} z_{2}^{2}-z_{3}\left(P_{n e t}+\frac{2 x_{3}}{c_{d c} R_{d c}}+\hat{P}_{s c}+\dot{x}_{3}^{*}\right)+\tilde{P}_{s c}\left(\frac{1}{\gamma} \tilde{P}_{s c}-z_{3}\right)$

Equation (37) suggests the following control law:

$P_{n e t}=c_{3} z_{3}-\frac{2 x_{3}}{C_{d c} R_{d c}}-\hat{P}_{s c}-\dot{x}_{3}^{*}$ 
And the following parameter adaptation law:

$\tilde{\tilde{P}}_{s c}=\gamma z_{3}$

In fact, substituting (38) and (39) in (37) yields:

$\dot{V}=-c_{1} z_{1}^{2}-c_{2} z_{2}^{2}-c_{3} z_{3}^{2}$

The outer closed loop equation is given by substituting (38) in (32). One obtains:

$\dot{z}_{3}=-c_{3} z_{3}-\tilde{P}_{s c}$

Now, as $P_{\text {net }}$ is a virtual control input, we make use of equation (34) to obtain the following control law:

$\left[\begin{array}{l}\overline{l_{f \alpha}} \\ \overline{l_{f \beta}}\end{array}\right]=\frac{1}{v_{f \alpha^{2}}+v_{f \beta}{ }^{2}}\left[\begin{array}{cc}v_{f \alpha} & -v_{f \beta} \\ v_{f \beta} & v_{f \alpha}\end{array}\right]\left[\begin{array}{c}P_{\text {net }} \\ 0\end{array}\right]$

Substituting (38) into (42) one finds the following expression of the fundamental current references $\left[\begin{array}{ll}\overline{l_{f \alpha}} & \overline{l_{f \beta}}\end{array}\right]^{T}$ :

$\left[\begin{array}{l}\overline{l_{f \alpha}} \\ l_{f \beta}\end{array}\right]=\frac{1}{v_{f \alpha^{2}+v_{f \beta}{ }^{2}}}\left[\begin{array}{l}v_{f \alpha}\left(c_{3} Z_{3}-\frac{2 x_{3}}{c_{d c} R_{d c}}-\widehat{\mathrm{P}}_{\mathrm{sc}}-\dot{x}_{3}^{*}\right) \\ v_{f \beta}\left(c_{3} Z_{3}-\frac{2 x_{3}}{c_{d c} R_{d c}}-\widehat{\mathrm{P}}_{\mathrm{sc}}-\dot{x}_{3}^{*}\right)\end{array}\right]$

\section{Simulation and discussion of results}

We now evaluate the new cascade regulator (referred to NonLinear-Magnetic Adaptive Control: NLM-AC) including the current control loop (26) and the adaptive voltage control loop ((43), (39)) Fig. 2. The simulation is performed using the SAPF structure depicted by Fig. 1. The nonlinear load is constituted by an AC-DC three-phase converter associated with an $R L$ load. The load and filter characteristics are summarized in Table 1.

\begin{tabular}{|c|c|c|}
\hline \multicolumn{3}{|c|}{ TABLE 1. SHUNT APF CHARACTERISTICS } \\
\hline PAR & & VALUES \\
\hline Power active filter & $\begin{array}{l}L_{f 0} \\
C_{d c} \\
R_{f} \\
\end{array}$ & $\begin{array}{c}20 \mathrm{mH} . \\
1000 \mu \mathrm{F} . \\
0,2 \Omega .\end{array}$ \\
\hline Rectifier-Load & $\begin{array}{l}L \\
R\end{array}$ & $\begin{array}{c}10 \mathrm{mH} . \\
100-10 \Omega\end{array}$ \\
\hline
\end{tabular}

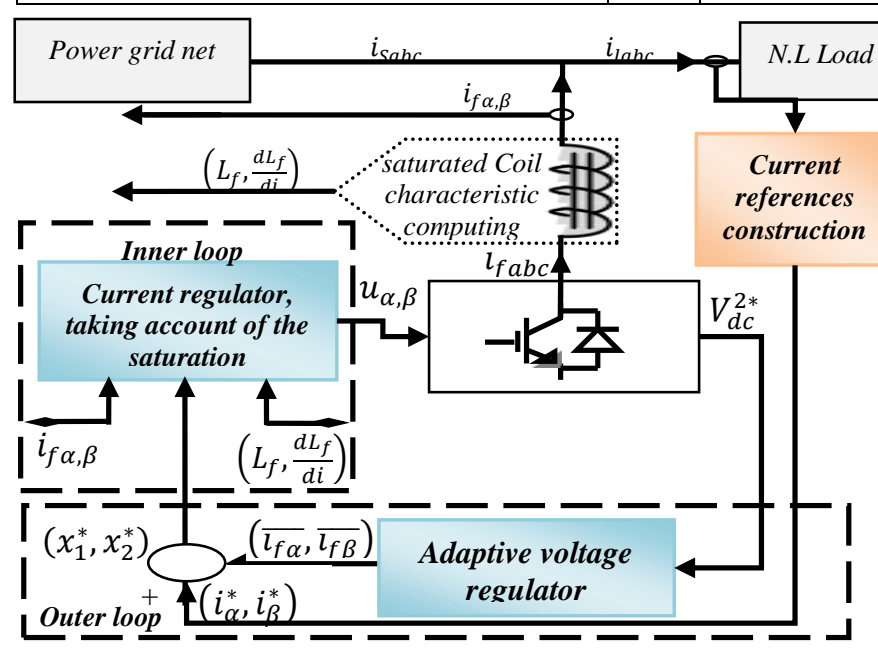

Fig. 2. Synoptic scheme of the cascade control strategy.

To evaluate the performances of the proposed regulator in different operation points, the load resistance is made variable; The resulting load current is illustrated by Fig. 3 . The strong harmonic distortion of this current is shown by Fig. 4. The magnetic flux through the coil depends on the current according to the law (1) and the corresponding magnetic characteristic $\phi=\lambda(i)$ is defined by Fig. 5. The resulting derivative $\frac{d L_{f}(.)}{d i}$ with respect to the inductor current is described by Fig.6.

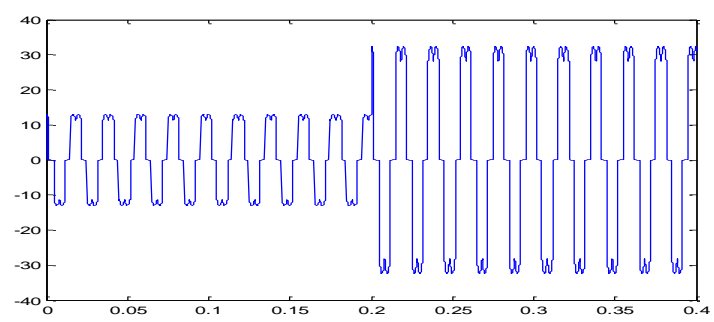

Fig.3 Line current before filtering in time domain

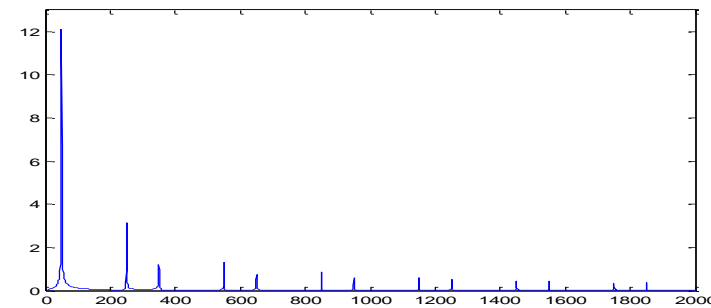

Fig.4: Load current in frequency domain.

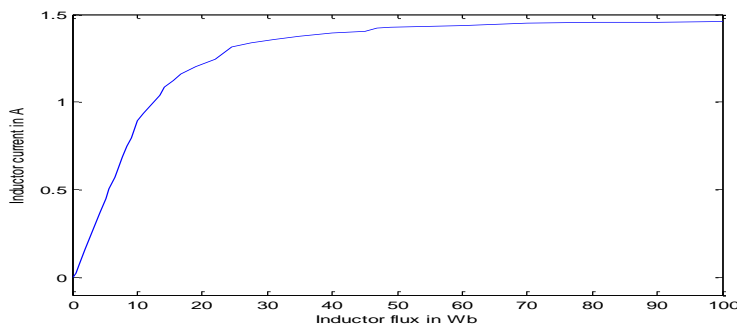

Fig. 5: Magnetic characteristic of the coil core

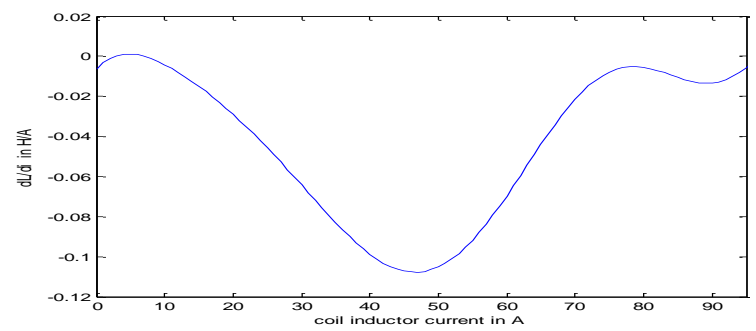

Fig. 6: Inductance derivative vs. inductor current

\section{NLM-AC Performance Evaluation}

The inner and the outer (NLM-AC) regulators are implemented using equations (26) and (36, 38). The corresponding design parameters are given the following numerical values of Table 3 , which proved to be convenient. In this respect, note that there is no systematic way, especially in nonlinear control, to make suitable choices for these values. Therefore, the usual practice consists in proceeding with trial-error approach. Doing so, the numerical values of Table 2 are retained.

\section{TABLE 2. NLM-AC CONTROLLER PARAMETERS}

\begin{tabular}{|l|l|l|}
\hline Current regulator & $c_{1}$ & $5.10^{3}$ \\
\cline { 2 - 3 } & $c_{2}$ & $5.10^{3}$ \\
\hline Voltage regulator & $c_{3}$ & 100 \\
& $\gamma$ & 10 \\
\hline
\end{tabular}

Recall that the (NLM-AC), is based on the model that take into account the magnetic saturation on the coil filter. The 
resulting controller performances are illustrated by Figs.7 to 10. Figs.7-8 show that independently of the load current level, the AC three-phases filter currents track well their references, confirming the theoretical results. The resulting network line current is plotted in Fig. 9, which shows that this current is clearly clean of harmonics, unlike the load current. This is better illustrated by Fig. 10 which shows the spectra of the load and net currents. It is seen that the net current is mainly constituted by a single component, situated in $50 \mathrm{~Hz}$. The higher frequency harmonics have well been suppressed.

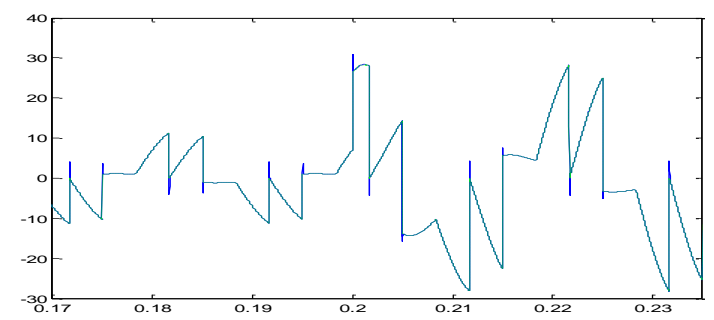

Fig.7: inner-loop tracking performances: $i_{f \alpha}$ current and its reference

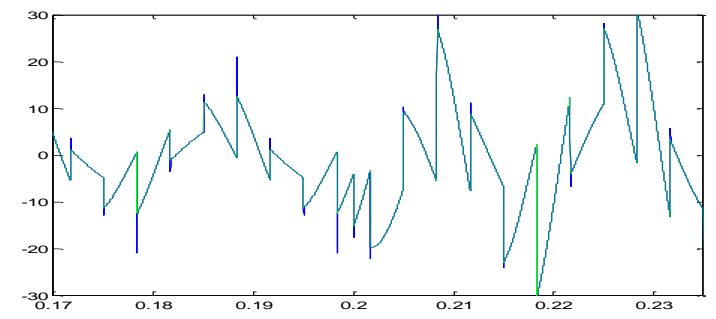

Fig.8: inner-loop tracking performances: $\mathrm{i}_{\mathrm{f} \beta}$ current and its reference

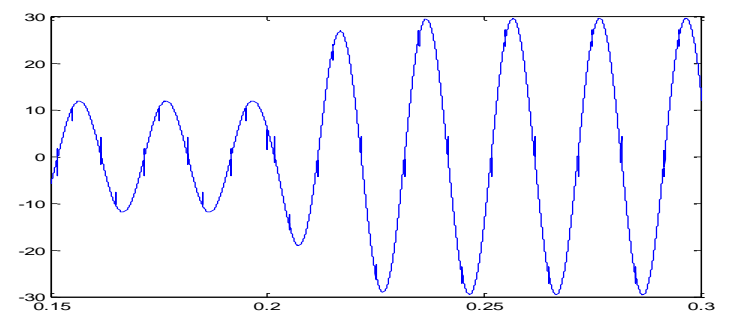

Fig.9: Network current shape with NLM-AC in time domain

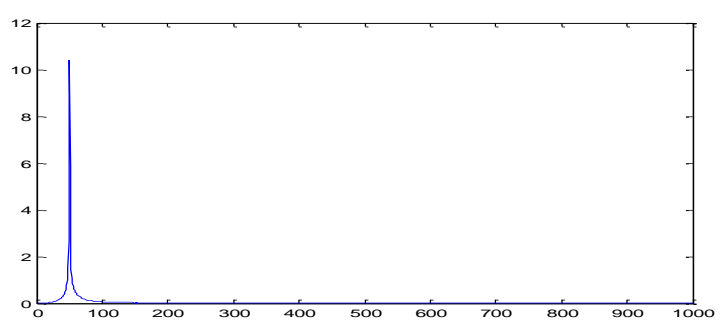

Fig.10: Network current with NLM-AC in frequency domain

\section{CONCLUSION}

We have considered the problem of controlling three-phase shunt active power filters when the operation conditions are such that the involved coils cannot be characterized by a constant inductance coefficient. This is particularly the case of high power coils operating in the presence of significantly changing conditions e.g. large variations of the converter load. The control objective is to achieve current harmonics and reactive power compensation, as well as tight voltage regulation at the inverter output capacitor. To this end, a new model of the three-phase SAPF is developed that accounts for the nonlinear nature of the coil magnetic characteristics. Using tools from the system averaging analysis theory, it is formally shown that the controller meets its objectives in the mean This formal result is confirmed by several simulations illustrating the performances of the proposed controller.

\section{REFERENCES:}

[1] Komurcugil, H. (2006) "A new control strategy for singlephase shunt active power filters using a Lyapunov function" IEEE Trans. on Industrial Electronics, vol. 53, pp. 305 - 312.

[2] Umamaheswari. M.G., G. Uma, (2013) "Analysis and design of reduced order linear quadratic regulator control for three phase power factor correction using cuk rectifiers" Electric Power Systems Research 96,1, 8

[3] Zaveri. N., A. Chudasama, (2012) "Control strategies for harmonic mitigation and power factor correction using shunt active filter under various source voltage conditions" International Journal of Electrical Power \& Energy Systems, 42, 661-671

[4] Panda A.K., S. Mikkili (2013) "FLC based shunt active filter $\left(p-q\right.$ and $\left.I_{d}-I_{q}\right)$ control strategies for mitigation of harmonics with different fuzzy MFs using MATLAB and real-time digital simulator" international journal of Electrical Power and Energy Systems 47 (2013) 313-336

[5] Bin Liu, Jia Ju Wu, Jun Li, Ji Yang Dai (2013) "A novel PFC controller and selective harmonics suppression" Electrical Power and Energy Systems 44, pp. 680-687

[6] Mikkili. S., A.K. Panda, (2012), "Real-time implementation of PI and fuzzy logic controllers based shunt active filter control strategies for power quality improvement" International Journal of Electrical Power \& Energy Systems, 43. 11141126

[7] Escobar, G., A.M. Stankovic, and P.Mattavelli, (2004). "An adaptive controller in stationary reference frame for $D$ statcom in unbalance operation” IEEE Trans. Ind. Electron., vol. 51, no. 2, pp. 401-409

[8] Rahmani, S., A.Hamadi, and K.Al-Haddad "A LyapunovFunction-Based Control for a Three-Phase Shunt Hybrid Active Filter" IEEE Transactions On Industrial Electronics, Vol. 59, No. 3, pages:1418-1429, 2012.

[9] Djerioui, A., K. Aliouane, F. Bouchafaa (2014) "Sliding mode direct power control strategy of a power quality based on a sliding mode observer" international journal of Electrical of Power and Energy Systems 56 (2014) 325-331

[10] Krein P.T., J. Bentsman, R.M. Bass, and B. Lesieutre (1990). "On the use of averaging for analysis of power electronic system" IEEE Trans. Power Electronics, vol. 5, pp. 182-190.

[11] Tse, C. K. and M. H. L. Chow (2000). "Theoretical study of switching converters with power factor correction and output regulation”. IEEE Trans. Circuits \& Systems I, vol. 47, pp. 1047-55.

[12] Subrahmanyam, V. (1994) "Representation of magnetization curves by exponential series", IEEE Proceedings, 121, no 7, pp. 707-708.

[13] Leonhard, W. (1996) "Control of Electrical Drives", Springer-Verlag, 2nd edition, NY

[14] Ouadi H., F.Giri, A.Elfadili, L.Dugard (2010). "Induction machine speed control with flux optimization" Control Engineering Practice 18.55-66.

[15] Levi, E. (1996) "Main flux saturation modelling in double cage" IEEE Transactions on Energy Conversion, Vol. 11 No 2, pp. 305-311

[16] Akagi, H., Y.Kanazawa, and A.Nabae, (1984) "Instantaneous reactive power compensators comprising switching devices without energy storage components" IEEE Trans. Industry Applications, vol. 20(3), pp. 625-630 